\title{
The effect of supplementation of Lactobacillus paracasei HIIO1 on salivary cortisol, and dehydroepiandrosterone sulfate (DHEA-S) levels
}

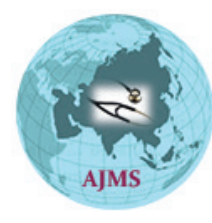

\author{
Ekasit Lalitsuradej ${ }^{1}$, Bhagavathi Sundaram Sivamaruthi², Sasithorn Sirilun ${ }^{3}$, \\ Phakkharawat Sittiprapaporn ${ }^{4}$, Sartjin Peerajan ${ }^{5}$, Chaiyavat Chaiyasut ${ }^{6}$
}

${ }^{1}$ Doctoral Student, ${ }^{2}$ Postdoctoral Research Fellow, ${ }^{3}$ Assistant Professor, ${ }^{5}$ Researcher, ${ }^{6}$ Assistant Professor and Head, Innovation Center for Holistic Health, Nutraceuticals and Cosmeceuticals, Faculty of Pharmacy, Chiang Mai University, Chiang Mai, Thailand, ${ }^{4}$ Assistant Professor and Head, Brain Science and Engineering Innovation Research Group, School of Anti-Aging and Regenerative Medicine, Mae Fah Luang University, Bangkok, Thailand, and Department of Anti-Aging Science, School of Anti-Aging and Regenerative Medicine, Mae Fah Luang University, Bangkok, Thailand

Background: Fatigue is one of the serious health issues, and stress is the main factor that induces chronic fatigue syndrome (CFS). The dysregulation of stress management pathway may account for the development of CFS. The human body comprises several neurobiological networks to manage physical and emotional insults. Hypothalamic-pituitary-adrenal (HPA) axis is one of the important neuroendocrine networks involved in the neurophysiological activity of the host system. The response of HPA axis depends on the physical and psychological state of stress and other factors like time and duration of stress. The probiotic supplements are proved as an adjuvant therapeutic agent for several diseases. Aims and Objective: The aim of the current study was to evaluate the effect of Lactobacillus paracasei HIIO1 supplementation on salivary cortisol and DHEA-S levels of fatigue subjects. Materials and Methods: After 12-weeks of probiotic intervention significantly reduced the salivary cortisol level, while DHEA-S level was not affected. The ratio of cortisol: DHEA-S was reduced after probiotic intervention. Results: The results concluded that L. paracasei $\mathrm{HIIO} 1$ has the ability to reduce the stress level in fatigue subjects by reducing the salivary cortisol level. Conclusion: The results evidently to recommend that the ingestion of 12.5 billion CFU of $L$. paracasei HIIO1 per day for 12 weeks significantly amended HPA-Axis by lowering salivary cortisol and ratio cortisol: DHEA-S in fatigue subjects.

Access this article online

Website:

http://nepjol.info/index.php/AJMS

DOI: 10.3126/ajms.v11i1.26500

E-ISSN: 2091-0576

P-ISSN: 2467-9100

Key words: Probiotics; Lactobacillus paracasei; Fatigue; Stress; Cortisol; HPA-Axis.

\section{INTRODUCTION}

Chronic fatigue syndrome (CFS) is one of the unbearable neurological diseases. ${ }^{1}$ Defects in memory, concentration, sleep pattern, muscular-skeletalpain, and constant fatigue are the common symptoms of CFS. ${ }^{2}$ The severity of the symptoms is unique among the patients, and some of the patients can manage their symptoms by restricting their energy needs. ${ }^{3}$ The underlying mechanism and cause of CFS were not yet elucidated.

The defect in regulation of stress management pathway may responsible for the development of CFS. ${ }^{1,3}$ Human body comprises several neurobiological networks to manage stress. Hypothalamic-pituitary-adrenal (HPA) axis is one of the important neurological axes having role in stress response, and maintaining the homeostasis. The response of HPA axis depends on the physical and psychological state of stress and other factors like time and duration of stress. ${ }^{4-7}$

The gut microbiota has direct influence on HPA axis and neuroendocrine system. ${ }^{8,9}$ The changes in the gut microbial composition will affect the psychological well-being, which is now considered as a complementary therapeutic approach to treat psychological disorders like depression 
and anxiety. ${ }^{10}$ The oral administration of potent microbes, generally probiotics, are proven as adjuvant therapeutic method for several diseases and disorders. ${ }^{11-16}$ It has been reported that the supplementation of probiotics improved the human mental health status. ${ }^{17}$

Cortisol is hormone, which helps coping stress, released by the adrenal glands with the response to corticotrophinreleasing factor. The stress hormone levels were increased up to two-five folds during stress. ${ }^{18}$ The adrenal glands also produce hormone dehydroepiandrosterone-S (DHEA-S). DHEA-S is believed as one of the stress-responsive hormones. The low level of DHEA-S associated with increased psychological illness and stress. ${ }^{19}$ The objective of the current study was to evaluate the effect of Lactobacillus paracasei HII01 supplementation on salivary cortisol and DHEA-S levels of fatigue subjects.

\section{MATERIALS AND METHODS}

Preparation and intervention of probiotics

The probiotic strain Lactobacillus paracasei HII01 was cultured and lyophilized at Lactomason Co., Ltd., South Korea. The live powdered probiotic strain was used for the study. The sachet containing $12.5 \times 10^{10} \mathrm{CFU} / \mathrm{g}$ of $\mathrm{L}$. paracase $\mathrm{HII} 01$ was given to volunteers and insisted them to consume for twelve weeks (one sachet per day).

Measurement of cortisol and DHEA-Sulfate level The saliva samples were collected from the volunteers at baseline and after twelve weeks of interventionat 7-9 am and stored at $4^{\circ} \mathrm{C}$ until use. The salivary cortisol and DHEA-S levels were measured by the ELISA method using The Eagle Biosciences Salivary Cortisol ELISA Assay kit as per the manufacturer's instructions.

\section{Statistical analysis}

The statistical program STATA15 was used for the data analysis. The Paired-sample t-test was used to distinguish within-group differences.

\section{RESULTS}

Demographic information of the subjects

Nine volunteers participated in the study. The mean age and BMI of the participants were $48.33 \pm 15.69$ years, and 27.91 $\pm 3.05 \mathrm{~kg} / \mathrm{m}^{2}$ at the time of study, respectively (Table 1 ).

\section{Changes in Salivary Cortisol, and DHEA-S levels}

The salivary cortisol level at baseline and after treatment was $202.78 \pm 84.32$ and $156.24 \pm 61.96 \mathrm{ng} / \mathrm{ml}$, respectively. The salivary cortisol level was significantly $(p=0.003)$ decreased, after the probiotic intervention. Whereas, the DHEA-S level was not affected by the probiotic intervention. The level of DHEA-S was $0.71 \pm 0.42$ and $0.71 \pm 0.45 \mathrm{ng} / \mathrm{ml}$ at baseline and after treatment, respectively (Table 2).

\section{Changes in Ratio of Cortisol: DHEA-S}

The ratio of cortisol: DHEA-S at baseline and after probiotic treatment was $460.61 \pm 409.30$, and $374.14 \pm 381.52 \mathrm{ng} / \mathrm{ml}$, respectively. The probiotic supplementation significantly $(p=0.011)$ reduced the ratio of cortisol: DHEA-S (Table 2).

\section{DISCUSSION}

The studies on the beneficial effect of probiotic supplementation on mental status of fatigue subjects are very limited. The current study revealed that the twelve-week supplementation of $L$. paracasei HII01 effectively reduced the salivary cortisol level (Table 2), which indicated that the stress level has been reduced after the probiotic intervention.

The previous studies reported that the consumption of L. belveticus R0052 and Bifidobacterium longum R0175 for thirty days reduced the urine cortisol level, ${ }^{10}$ and the strain L. gasseri CP2305 reduced the salivary cortisol level after 4 weeks of consumption. ${ }^{20}$

There was no change in the DHEA-S level in fatigue subjects after twelve-weeks of L. paracasei HII01 supplementation. The ratio of cortisol: DHEA-S was reduced after the probiotic intervention (Table 2).

\section{Table 1: Demographic Information of the} Subjects Participated in the Study

\begin{tabular}{lc}
\hline Characteristic & Number (Percentage) \\
\hline Gender & \\
Male & $4(44.4)$ \\
Female & $5(55.6)$ \\
Age (Years) & \\
$\leq 30$ & $2(22.2)$ \\
$31-59$ & $4(44.5)$ \\
60 or more & $3(33.3)$ \\
Total (Mean \pm SD) & $9(48.33 \pm 15.69)$ \\
BMl & \\
$<25$ & $2(22.2)$ \\
$\geq 25$ & $7(77.8)$ \\
Total (Mean \pm SD) & $9(27.91 \pm 3.05)$ \\
\hline
\end{tabular}

\begin{tabular}{|c|c|c|c|}
\hline Biomarkers & $\begin{array}{c}\text { Baseline } \\
\text { (ng/ml) }\end{array}$ & $\begin{array}{c}\text { After } 12 \\
\text { weeks (ng/ml) }\end{array}$ & $p$-value \\
\hline $\begin{array}{l}\text { Salivary } \\
\text { Cortisol }\end{array}$ & $202.78 \pm 84.32$ & $156.24 \pm 61.96$ & 0.003 \\
\hline $\begin{array}{l}\text { Salivary } \\
\text { DHEA-S }\end{array}$ & $0.71 \pm 0.42$ & $0.71 \pm 0.45$ & 1.00 \\
\hline $\begin{array}{l}\text { Cortisol } \\
\text { :DHEA-S ratio }\end{array}$ & $460.61 \pm 409.30$ & $\begin{array}{c}374.14 \pm \\
381.52\end{array}$ & 0.011 \\
\hline
\end{tabular}


The beneficial effect of probiotic supplementation depends on the strain, concentration, duration, and other aided activities. The single strain of probiotic L. gasseri CP2305 showed positive effects on stress management, ${ }^{20}$ and multistrain probiotic supplementation (L. helveticus $\mathrm{R} 0052$ and B. longum R0175) also reduced the stress. ${ }^{10}$

The current study was an initial clinical trial on the effect of $L$. paracasei HII01 on stress management in fatigue subjects. It has some disadvantages such as very limited number of subjects, limited analyzed parameters, no placebo control, and subjects with different lifestyles. The further extended study is needed to uncover the favorable impact of L. paracase $i$ HII01 on stress management in fatigue subjects.

\section{CONCLUSION}

The results evidently to recommend that the ingestion of 12.5 billion CFU of L. paracasei $\mathrm{HII} 01$ per day for 12 weeks significantly amended HPA-Axis by lowering salivary cortisol and ratio cortisol: DHEA-S in fatigue subjects. However, the results of the current study need to be validated with an increased number of subjects and placebo control since the present study has some limitations.

\section{ACKNOWLEDGEMENT}

The authors would like to acknowledge the financial support from Center of Excellence on Medical Biotechnology (CEMB), The S\&T Postgraduate Education and Research Development Office (PERDO), The Commission on Higher Education (CHE), Thailand. Authors gratefully acknowledge Chiang Mai University grant (CMU-grant) for the support.Authors also gratefully acknowledge Brain Science and Engineering Innovation Research Group, Mae Fah Luang University grant (2019) (MFU-grant no. 611U109005) and 2020, Thailand for the support.

\section{REFERENCES}

1 Chaudhuri A and Behan PO. Neurological dysfunction in chronic fatigue syndrome. J Chronic Fatigue Syndr 2000; 6: 51-68.

2. Atkinson HC, Leggett JD, Wood SA, Castrique ES, Kershaw YM and Lightman SL. Regulation of the hypothalamicpituitary-adrenal axis circadian rhythm by endocannabinoids is sexually diergic. Endocrinology 2010; 151: 3720-3727.
3. Van Houdenhove B, Van Den Eede F and Luyten P. Does hypothalamic-pituitary-adrenal axis hypofunction in chronic fatigue syndrome reflect a 'crash' in the stress system?. Med Hypotheses 2009; 72: 701-705.

4. Smith SM and Vale WW. The role of the hypothalamicpituitaryadrenal axis in neuroendocrine responses to stress. Dialogues Clin Neurosci 2006; 8: 383-395.

5. Elenkov IJ and Chrousos GP. Stress system: organization, physiology and immunoregulation. Neuroimmunomodulation, 2007; 13: 257-267.

6. Lanfumey L, Mongeau R, Cohen-Salmon C and Hamon M. Corticosteroid-serotonin interactions in the neurobiological mechanisms of stress-related disorders. Neurosci Biobehav Rev 2008; 32: 1174-1184.

7. Kudielka BM, Schommer NC, Hellhammer DH and Kirschbaum C. Acute HPA axis responses, heart rate, and mood changes to psychosocial stress (TSST) in humans at different times of day. Psychoneuroendocrinology 2004; 29: 983- 992.

8. Sudo N. Microbiome, HPA axis and production of endocrine hormones in the gut. Adv Exp Med Biol 2014; 817: 177-194.

9. Farzi A, Fröhlich EE and Holzer P. Gut Microbiota and the Neuroendocrine System. Neurotherapeutics 2018; 15: 5-22.

10. Messaoudi M, Violle N, Bisson JF, Desor D, Javelot $H$ and Rougeot C. Beneficial psychological effects of a probiotic formulation (Lactobacillus helveticus R0052 and Bifidobacterium longum R0175) in healthy human volunteers. Gut Microbes 2011; 2: 256-261.

11. Blaabjerg S, Artzi DM and Aabenhus R. Probiotics for the prevention of antibiotic-associated diarrhea in outpatients-a systematic review and meta-analysis. Antibiotics (Basel) 2017; 6: 21.

12. Sivamaruthi BS. A comprehensive review on clinical outcome of probiotic and synbiotic therapy for inflammatory bowel diseases. Asian Pac J Trop Biomed 2018; 8: 179-186.

13. Sivamaruthi $B S$, Kesika $P$ and Chaiyasut $C$. Influence of probiotic supplementation on climacteric symptoms in menopausal women-A mini review. Int J App Pharm 2018; 10: 43-46.

14. Sivamaruthi BS, Kesika $P$ and Chaiyasut $C$. Probiotic based therapy for atopic dermatitis: Outcomes of clinical studies. Asian Pac J Trop Biomed 2018; 8: 328-332.

15. Sivamaruthi BS, Kesika $P$ and Chaiyasut $C$. A review on antiaging properties of probiotics. Int J App Pharm 2018; 10: 23-27.

16. Sivamaruthi $B S$, Kesika $P$, Suganthy $N$ and Chaiyasut $C$. A review on role of microbiome in obesity and antiobesity properties of probiotic supplements. Biomed Res Int 2019; 2019: 3291367.

17. Sivamaruthi BS, Prasanth MI, Kesika $P$ and Chaiyasut C. Probiotics in human mental health and diseases-A mini review. Trop J Pharm Res 2019; 18: 889-895.

18. Ranabir $\mathrm{S}$ and Reetu K. Stress and hormones. Indian J Endocrinol Metab. 2011; 15: 18-22.

19. Lennartsson AK, Theorell T, Rockwood AL, Kushnir MM and Jonsdottir IH. Perceived stress at work is associated with lower levels of DHEA-S. PloS one 2013; 8: e72460.

20. Nishimura M, Ohkawara T, Tetsuka K, Kawasaki Y, Nakagawa R, Satoh $\mathrm{H}$, et al. Effects of yogurt containing Lactobacillus plantarum HOKKAIDO on immune function and stress markers. J Tradit Complement Med 2016; 6: 275 -280. 


\section{Authors Contribution:}

EL- Concept and design of the study, statistically analyzed and interpreted, manuscript preparation; BSS- Statistically analyzed and interpreted, manuscript preparation, critical revision of the manuscript; SS- Concept and design of the study, statistically analyzed and interpreted; PS- Manuscript preparation, critical revision of the manuscript; SP-Concept and design of the study; CC-Concept and design of the study, statistically analyzed and interpreted, manuscript preparation, critical revision of the manuscript

\section{Work attributed to:}

Innovation Center for Holistic Health, Nutraceuticals and Cosmeceuticals, Faculty of Pharmacy, Chiang Mai University, Thailand;

Brain Science and Engineering Innovation Research Group, School of Anti-Aging and Regenerative Medicine, Mae Fah Luang University, Thailand.

\section{Orcid ID:}

Mr. Ekasit Lalitsuradej- https://orcid.org/0000-0002-8615-9412

Dr. Bhagavathi Sundaram Sivamaruthi- iD https://orcid.org/0000-0002-5499-8350

Dr. Sasithorn Sirilun-10 https://orcid.org/0000-0002-1508-2358

Dr. Phakkharawat Sittiprapaporn- (D) https://orcid.org/0000-0002-4103-9396

Mr. Sartjin Peerajan-10 https://orcid.org/0000-0002-0995-3401

Dr. Chaiyavat Chaiyasut-@ https://orcid.org/0000-0002-1633-2419

Source of Support: Center of Excellence on Medical Biotechnology (CEMB), The S\&T Postgraduate Education and Research Development Office (PERDO); The Commission on Higher Education (CHE), Thailand; Chiang Mai University grant (CMU-grant), Thailand; Brain Science and Engineering Innovation Research Group, Mae Fah Luang University grant (2019) (MFU-grant no. 611U109005) and 2020, Thailand, Conflict of Interest: None. 